\title{
ORIGINAL
}

\section{EVALUACIÓN DE UN SISTEMA DE DEVOLUCIÓN DE INFORMACIÓN EN SALUD PÚBLICA: EXPERIENCIA EN EL ÁREA 2 DE LA COMUNIDAD DE MADRID}

\author{
Pedro Arias Bohigas (1), Karoline Fernández de la Hoz (2), Felicitas Domínguez Berjón (2), Rosa \\ Noguerales de la Obra (2), Dionisio Herrera Guibert (3) y Ferrán Martínez Navarro (3) \\ (1) Programa de Epidemiología Aplicada de Campo (PEAC). Centro Nacional de Epidemiología. Instituto de Sa- \\ lud Carlos III \\ (2) Servicio de Salud Pública del Área 2. Dirección General de Salud Pública. Consejería de Sanidad. Comunidad \\ de Madrid \\ (3) Centro Nacional de Epidemiología. Instituto de Salud Carlos III
}

\section{RESUMEN}

Fundamento: En enero del año 2000 el Servicio de Salud Pública del Área Sanitaria 2 de la Comunidad de Madrid, inició la edición semanal de una Hoja de Información en Salud Pública (HISP) dirigida a los profesionales de la salud del Área. El presente trabajo tiene como objetivo estimar el conocimiento, aceptabilidad, utilidad y difusión de la HISP durante el año 2000.

Métodos: Se realizó una encuesta por correo a una muestra de profesionales sanitarios del Área 2. Tras un análisis descriptivo se investigó, mediante análisis multivariante, qué factores explicaban que un profesional del Área conociera la HISP. Se utilizó el programa Epi Info 2000.

Resultados: La tasa de respuesta fue del 79,7\%. El apartado mejor valorado fue el de alertas en salud pública. Se encontró una asociación entre conocer la HISP y conocer el Boletín Epidemiológico de la Comunidad de Madrid ( $\mathrm{OR}=9,3$, IC $95 \%=2,9-29,5)$, y entre ser médico y recibir información de salud pública $(\mathrm{OR}=4,3$, IC: $1,5-12,6, \mathrm{p}=0,005)$.

Conclusiones: La HISP ha tenido buena aceptación entre los profesionales sanitarios del Área 2 que valoran más los contenidos relacionados con situaciones estresantes. Es necesario mejorar la difusión de la información de salud pública entre los profesionales de enfermería.

Palabras clave: Publicaciones periódicas. Salud Pública. Vigilancia. Estudios de evaluación.

Correspondencia:

Pedro Arias Bohigas

C/ Chantada, 41

28034 Madrid

Correo electrónico: p.arias@madrid.org

\section{ABSTRACT}

\section{Evaluation of a System to Feedback Information on Public Health: The experience of Area 2 of the Community of Madrid}

Background: In January 2000 the Public Health Service of Area 2, in the Region of Madrid, began to publish a weekly sheet on epidemiological and public health information (HISP) addressed to health professionals working in the Area. The aim of the present study was to estimate to how extend the «HISP» was known among health professionals of Area 2, and also to estimate the suitability and usefulness of its contents, during 2000.

Methods: A postal survey was carried out among a random sample of professionals working in Area 2. A descriptive analysis of main variables was done, variables associated to the knowledge of the «HISP» were investigated. Epi Info 2000 program was used for the analysis.

Results: The response rate was $79,7 \%$. The best evaluated content was that related to epidemic outbreaks. An association between knowing the «HISP» and knowing the regional epidemiological bulletin (Boletín Epidemiológico de la Comunidad de Madrid) was found (OR=9,3, IC 95\%=2,9-29,5), and also an association between knowing the «HISP» and being a physician $(\mathrm{OR}=4,3$, IC: $1,5-12,6, \mathrm{p}=0,005)$.

Conclusions: The «HISP» has a good acceptance among health professionals of Area 2. They were more interested in contents related to stressful situations. It is necessary to improve the diffusion of information on public health among nursing professionals.

Key words: Periodicals. Public health. Surveillance. Evaluation studies. 


\section{INTRODUCCIÓN}

La clásica definición de A Langmuir sobre el concepto de vigilancia epidemiológica (1962) incluye la frase: «Intrínseca al concepto es la distribución de los datos básicos y su interpretación, a todos aquellos que han contribuido y a todos los que necesitan conocerlos» ${ }^{1}$. La devolución de información es una de las tareas fundamentales de todo sistema de vigilancia epidemiológica moderno $^{2,3}$. En España, desde hace varias décadas, existen medios para la difusión de información epidemiológica y de salud pública como parte de los sistemas de vigilancia, tanto a nivel estatal (Boletín Epidemiológico Semanal) como autonómico (Boletines Epidemiológicos de Comunidades Autónomas). En la Comunidad de Madrid, desde 1990 se publica el Boletín Epidemiológico de la Comunidad de Madrid (BECM) ${ }^{4}$. Además, algunas áreas de salud publican boletines que difunden informes sobre temas específicos (EDO, registro de tuberculosis, etc.) con distinta periodicidad.

Por otra parte, la mayor accesibilidad a Internet en los últimos años ha incrementado la presencia en la red de información sobre salud pública asociada a sistemas de vigilancia. Este medio, por sus características de inmediatez y facilidad de difusión, se está constituyendo en una herramienta potencial de gran utilidad ${ }^{5}$. Así, por ejemplo, la Junta de Andalucía está usando el correo electrónico como medio de difusión de información de salud pública. La necesidad de ofrecer información útil y oportuna a las personas que deben conocerla, y la mayor facilidad de edición y distribución hacen previsible un desarrollo importante de los mecanismos de devolución de información. Será necesario, por tanto, profundizar en las características que deben cumplir y la evaluación que se puede realizar de los mismos. Sin embargo, mientras que la evaluación de los sistemas de vigilancia epidemiológica se ve como una actividad necesaria, lo que queda reflejado en numerosas publicaciones ${ }^{7-9}$, desconocemos la existencia de traba- jos en los que, de una forma explícita, se evalúe la devolución de la información producida por uno de estos sistemas a los profesionales que la generaron.

En enero del año 2000 los Servicios de Salud Pública de las Áreas Sanitarias 2 y 5 de la Comunidad de Madrid iniciaron la difusión de información en salud pública en su territorio de influencia (402.265 y 605.928 habitantes respectivamente). Puesto que para dar cumplimiento a sus objetivos los sistemas de vigilancia deben ser sencillos, flexibles, aceptables, oportunos y tan baratos como sea posible ${ }^{6}$, se consideró que estos mismos parámetros eran aplicables a la difusión de la información. Por ello se diseño una Hoja de Información en Salud Pública (HISP) de periodicidad semanal que se distribuye por fax, correo normal y correo electrónico entre los profesionales de salud de ambas Áreas Sanitarias. En ella se incluye información sobre cinco grandes temas: El Sistema de Enfermedades de Declaración Obligatoria (EDO), la enfermedad meningocócica (EM), la evolución de la temporada de gripe (TG), alertas en Salud Pública (AL) y otras noticias relacionadas con la Salud Pública $(\mathrm{ON})$.

Después de un año de funcionamiento se consideró necesario valorar el impacto y aceptación de la HISP.

El presente trabajo tiene como objetivos estimar el grado de conocimiento de dos sistemas de devolución de información (la HISP del Área 2 y el BECM) entre los profesionales sanitarios del Área 2 de la Comunidad de Madrid y evaluar la aceptabilidad, utilidad y difusión que la de la HISP tuvo durante el año 2000.

\section{SUJETOS Y MÉTODOS}

La población diana del estudio fueron las 593 personas receptoras de la HISP del Área 2 , constituida por diplomados universitarios de enfermería (DUE) y facultativos de atención primaria, facultativos especialistas hos- 
pitalarios en servicios de interés y técnicos y gestores del Área Sanitaria. Se seleccionó una muestra aleatoria simple para una prevalencia estimada de conocimiento de la de la HISP de un $85 \%$, aceptando un error máximo de un $6 \%$ y un nivel de confianza (1-alfa) de 0,95. El marco muestral fue el listado de profesionales proporcionado por la Gerencia de Atención Primaria de Insalud del Área 2 y el listado de profesionales notificadores de Enfermedades de Declaración Obligatoria del Servicio de Salud Pública del Ârea 2. El tamaño de la muestra fue de 118 individuos. Se realizó una encuesta por correo mediante un cuestionario autoadministrado diseñado específicamente para este fin. Los cuestionarios se acompañaban de una carta explicativa del estudio y de un sobre franqueado con la dirección de destino ya impresa. La encuesta se realizó entre los días 28 de enero y 15 de febrero de 2001. En caso de no recibir respuesta espontánea se solicitaba la remisión del cuestionario de forma telefónica hasta por dos veces. En todo el proceso se mantuvo la confidencialidad de los datos de los entrevistados.

En el cuestionario se incluyeron preguntas referentes a las características personales de los entrevistados, y otras destinadas a valorar la difusión de la HISP y el BECM, la aceptabilidad de la HISP, su utilidad y la oportunidad de sus contenidos. En estos parámetros se recoge la percepción de los receptores de la información; se trata por tanto de una valoración subjetiva. La oportunidad se valoró directamente utilizando una pregunta en la que el receptor calificaba la información como oportuna o no oportuna. $\mathrm{La}$ utilidad fue evaluada a través de dos preguntas: Utilidad para la mejora del conocimiento y utilidad para la toma de decisiones en su ámbito de trabajo. Respecto a la aceptabilidad, se incluyeron tres preguntas: una relativa a la frecuencia de consulta de la información incluida en la HISP, otra relativa al interés por los contenidos de la misma y una última sobre el uso de la HISP en sesiones clínicas, reuniones de trabajo, etc. Se realizó un análisis descriptivo de las princi- pales variables incluidas en el cuestionario. Para comparar proporciones y medias se usó el programa Epi Info 2000. Se realizó un análisis multivariante para determinar qué variables explicaban que un profesional del Área 2 conociera la HISP. Para este análisis se utilizó el módulo de Regresión Logística de Epi Info 2000.

\section{RESULTADOS}

Se recibió contestación al cuestionario de 94 personas, lo que corresponde a una tasa de respuesta del 79,7\%. La edad media de los que respondieron fue de 43,5 años $(\mathrm{DE}=9,5)$; el $65,9 \%$ eran mujeres; el $43,5 \%$ eran DUE y el $48,9 \%$ médicos de atención primaria. No se encontraron diferencias significativas en cuanto a la distribución por sexo y categoría profesional entre los encuestados que contestaron y la población de profesionales sanitarios del área. Tal como se muestra en la tabla 1 , conocían la HISP el $60,6 \%$ de los entrevistados y el $66,3 \%$ conocían el BECM. Entre los que conocían la HISP el 92,7\% declaraban consultarla alguna vez o con frecuencia, mientras que para el BECM este dato fue del $73,3 \%$. El $83,6 \%$ de los entrevistados que conocían la HISP, calificaron la información ofrecida como oportuna y el $66,0 \%$ consideraron que la periodicidad semanal era la más adecuada. Cuando se les preguntó si habían comentado alguna vez los contenidos de la HISP en alguna reunión de equipo de trabajo o sesión clínica el 56,6\% respondió afirmativamente. En la tabla 2 se presentan los resultados del interés expresado por los temas contenidos en la HISP, y la valoración de la utilidad que los mismos tienen para el conocimiento de la situación epidemiológica del Área, y para la toma de decisiones en el trabajo. Con una escala de 0 (valor más bajo) a 5, el ítem mejor valorado fue el apartado de Alertas en Salud Pública, tanto respecto al interés suscitado (puntuación media=4,2, DE=1,1), como para la ayuda al conocimiento de la situación del Área (media=3,8, DE=1,3) y para 
Tabla 1

Distribución de las personas encuestadas según las variables relativas al conocimiento de la Hoja de Información en Salud Pública del Área 2 de la Comunidad de Madrid (HISP) y el Boletín Epidemiológico de la Comunidad de Madrid (BECM)

\begin{tabular}{|c|c|c|c|c|c|c|}
\hline & \multicolumn{2}{|c|}{ Área $2(N=94)$} & \multicolumn{2}{|c|}{ D.U.E. $(N=37)$} & \multicolumn{2}{|c|}{ Médicos $(N=47)$} \\
\hline & $n / R V$ & & $n / R V$ & & $n / R V$ & \\
\hline Edad media (Desviación Típica) & $86 / 86$ & $43,5(9,5)$ & $37 / 37$ & $46,4(7,9)$ & $44 / 44$ & $46,3(10,3)$ \\
\hline Sexo: Mujer & $88 / 94$ & $65,9 \%$ & $36 / 37$ & $89,7 \%$ & $44 / 47$ & $47,7 \%$ \\
\hline Conoce la Hoja ISP & $57 / 94$ & $60,6 \%$ & $18 / 40$ & $45,0 \%$ & $33 / 47$ & $70,2 \%$ \\
\hline Conoce el BECM & $61 / 92$ & $66,3 \%$ & $21 / 40$ & $52,5 \%$ & $35 / 45$ & $77,8 \%$ \\
\hline \multirow{2}{*}{ Sobre los que conocen la Hoja ISP } & \multicolumn{2}{|c|}{ Área $2(N=57)$} & \multicolumn{2}{|c|}{ D.U.E. $(N=18)$} & \multicolumn{2}{|c|}{ Médicos $(N=33)$} \\
\hline & $n / R V$ & $\%$ & $n / R V$ & $\%$ & $n / R V$ & $\%$ \\
\hline \multicolumn{7}{|l|}{ Consulta la HISP (en los últimos 12 meses) } \\
\hline No & $4 / 55$ & 7,3 & $3 / 17$ & 17,6 & $1 / 32$ & 3,1 \\
\hline Alguna vez & $33 / 55$ & 60,0 & $10 / 17$ & 58,8 & $21 / 32$ & 65,6 \\
\hline Con frecuencia & $18 / 55$ & 32,7 & $4 / 17$ & 23,5 & $10 / 32$ & 31,3 \\
\hline \multicolumn{7}{|l|}{ Considera la HISP } \\
\hline Oportuna & $46 / 55$ & 83,6 & $15 / 18$ & 83,3 & $6 / 31$ & 80,6 \\
\hline Atrasada & $9 / 55$ & 16,4 & $3 / 18$ & 16,7 & $25 / 31$ & 19,4 \\
\hline \multicolumn{7}{|l|}{ Periodicidad ideal de la HISP } \\
\hline Semanal & $33 / 50$ & 66,0 & $10 / 17$ & 58,8 & $20 / 27$ & 66,7 \\
\hline Quincenal & $9 / 50$ & 18,0 & $3 / 17$ & 17,6 & $3 / 27$ & 11,1 \\
\hline Mensual & $7 / 50$ & 14,0 & $4 / 17$ & 23,5 & $3 / 27$ & 11,1 \\
\hline Trimestral & $1 / 50$ & 2,0 & $0 / 17$ & 0,0 & $1 / 27$ & 3,7 \\
\hline $\begin{array}{l}\text { Si ha comentado la HISP en alguna reunión } \\
\text { ¿Cómo recibe la HISP? }\end{array}$ & $30 / 53$ & 56,6 & $10 / 18$ & 55,6 & $16 / 29$ & 55,2 \\
\hline Por correo & $27 / 57$ & 47 & $7 / 18$ & 38,9 & $15 / 33$ & 45,5 \\
\hline Copia & $16 / 57$ & 28 & $6 / 18$ & 33,3 & $10 / 33$ & 30,3 \\
\hline Se ve en una reunión & $4 / 57$ & 7 & $2 / 18$ & 11,1 & $2 / 33$ & 6,1 \\
\hline Tablón de anuncios & $10 / 57$ & 18 & $3 / 18$ & 16,7 & $7 / 33$ & 21,2 \\
\hline \multirow{2}{*}{ Sobre los que conocen el BECM } & \multicolumn{2}{|c|}{ Area $2(N=61)$} & \multicolumn{2}{|c|}{ D.U.E. $(N=21)$} & \multicolumn{2}{|c|}{$\operatorname{Médicos}(N=35)$} \\
\hline & $n / R V$ & $\%$ & $n / R V$ & $\%$ & $n / R V$ & $\%$ \\
\hline \multicolumn{7}{|l|}{ Consulta el BECM (en los últimos 12 meses) } \\
\hline No & $16 / 60$ & 26,7 & $4 / 21$ & 19,1 & $6 / 34$ & 17,7 \\
\hline Alguna vez & $41 / 60$ & 68,3 & $10 / 21$ & 47,6 & $18 / 34$ & 52,9 \\
\hline Con frecuencia & $3 / 60$ & 5,0 & $1 / 21$ & 4,8 & $2 / 34$ & 5,9 \\
\hline
\end{tabular}

$\mathrm{N}=$ Número total de respuestas posibles; $\mathrm{RV}=$ Número de respuestas válidas; $\mathrm{n}=$ Número de respuestas afirmativas o relativas a la categoría de la fila.

la ayuda a la toma de decisiones en el ámbito de trabajo (media=3,3, DE=1,6). Cuando se compararon estas valoraciones entre DUE y médicos, no se encontraron diferencias estadísticamente significativas. Se investigaron las variables asociadas al conocimiento de la Hoja ISP, y en el análisis crudo (tabla 3 ) se encontró que los hombres conocían la Hoja ISP más que las mujeres $(\mathrm{OR}=2,8$, IC $95 \%=1,0-7,8)$; los médicos más que los DUE $(\mathrm{OR}=2,9$, IC $95 \%=1,2-6,9) ;$ y los que conocían el BECM más que los que no lo conocían $(\mathrm{OR}=10,0, \mathrm{IC} 95 \%=3,3-30,1)$. Se observó también que el $86,7 \%$ de los médicos recibían información epidemiológica: el 60,0\% 
Tabla 2

Valoración por los encuestados de los distintos apartados de la Hoja de Información en Salud Pública del Área 2 de la Comunidad de Madrid (HISP). Media y desviación estándar (DE) (N=57)

\begin{tabular}{|c|c|c|c|c|c|c|c|c|c|}
\hline \multirow{2}{*}{ Apartado } & \multicolumn{3}{|c|}{ Interés $^{I}$} & \multicolumn{3}{|c|}{ Ayuda al conocimiento ${ }^{2}$} & \multicolumn{3}{|c|}{$\begin{array}{c}\text { Ayuda a la toma } \\
\text { de decisiones }^{3}\end{array}$} \\
\hline & $n$ & Media & $D S$ & $n$ & Media & $D S$ & $n$ & Media & $D S$ \\
\hline Enfermedades de Declaración Obligatoria & 54 & 3,7 & $(1,2)$ & 54 & 3,6 & $(1,4)$ & 51 & 2,9 & $(1,6)$ \\
\hline Alertas en salud pública & 54 & 4,2 & $(1,1)$ & 52 & 3,8 & $(1,3)$ & 50 & 3,3 & $(1,6)$ \\
\hline Enfermedad meningocócica & 54 & 4,0 & $(1,0)$ & 55 & 3,7 & $(1,4)$ & 51 & 3,0 & $(1,6)$ \\
\hline Temporada de gripe & 54 & 3,4 & $(1,4)$ & 53 & 3,5 & $(1,4)$ & 46 & 2,6 & $(1,6)$ \\
\hline Otras noticias & 39 & 3,5 & $(1,4)$ & 47 & 3,0 & $(1,4)$ & 46 & 3,5 & $(1,4)$ \\
\hline
\end{tabular}

Interés del encuestado en el item de referencia.

2 Ayuda de la información de la HISP al conocimiento de la situación epidemiológica del área.

${ }_{3}^{3}$ Ayuda de la información de la HISP a la toma de decisiones en su ámbito de trabajo. Medias sobre una escala de 0 a 5 , donde 0 es la peor valoración posible. $\mathrm{n}=$ Número de respuesta válidas.

de ellos los dos boletines (BECM y HISP) y el $26,7 \%$ sólo recibía uno. Únicamente el $13,3 \%$ no recibía ninguno de los dos boletines. Entre los DUE un 37,5\% recibía los dos boletines, un $22,5 \%$ sólo recibía uno, y un $40,0 \%$ no recibía ninguno (tabla 4 ). Se encontró una asociación estadísticamente significativa entre ser médico y recibir in- formación epidemiológica —al menos uno de los dos boletines- $\quad(\mathrm{OR}=4,3$, IC:1,5-12,6, $\mathrm{p}=0,005)$. Al incluir las variables del estudio en un modelo de regresión logística, la única que permaneció como explicativa para el conocimiento de la HISP fue el conocimiento del BECM $(\mathrm{OR}=9,3$, IC $95 \%=2,9-29,5)$ (Tabla 3).

Tabla 3

Variables que influyen en el conocimiento de la Hoja de Información en Salud Pública del Área 2 de la Comunidad de Madrid Odds ratios (OR) crudos y ajustados en un modelo de regresión logística, intervalos de confianza al $95 \%$ y valor de p

\begin{tabular}{|l|c|c|c|c|c|c|}
\hline & OR crudo & I.C 95\% & $p$ & OR ajustado & IC 95\% & $P$ \\
\hline $\begin{array}{l}\text { Sexo } \\
\text { Mujer* } \\
\text { Varón }\end{array}$ & 1,0 & & & & & \\
\hline $\begin{array}{l}\text { Conoce el BECM } \\
\text { No* } \\
\text { Si }\end{array}$ & 2,8 & $1,0-7,8$ & 0,05 & 2,6 & $0,6-11,7$ & 0,18 \\
\hline $\begin{array}{l}\text { Grupo Profesional } \\
\text { DUE * }\end{array}$ & 1,0 & & & & & \\
Médico & 10,0 & $3,3-30,1$ & $<0,001$ & 9,3 & $2,9-29,5$ & $<0,001$ \\
\hline Edad & 1,0 & & & & & \\
\end{tabular}

* Nivel de referencia. 
Distribución de los profesionales encuestados que respondieron según el número de fuentes de las que reciben información epidemiológica - Hoja de Información en Salud Pública del Área 2 de la Comunidad de Madrid y Boletín Epidemiológico de la Comunidad de Madrid

\begin{tabular}{|l|c|r|r|r|r|r|}
\hline \multirow{2}{*}{ Número de fuentes } & \multicolumn{2}{|c|}{ Total Área } & \multicolumn{2}{c|}{ DUE } & \multicolumn{2}{c|}{ Médicos } \\
\cline { 2 - 7 } & $n$ & $\%$ & $n$ & $\%$ & \multicolumn{1}{c|}{$n$} & $\%$ \\
\hline Ninguna & 22 & 25,9 & 16 & 40,0 & 6 & 13,3 \\
Una (HISP o BECM) & 21 & 24,7 & 9 & 22,5 & 12 & 26,7 \\
Ambas (HISP y BEMC) & 42 & 49,4 & 15 & 37,5 & 27 & 60,0 \\
\hline Total & 83 & 100,0 & 40 & 100,0 & 43 & 100,0 \\
\hline
\end{tabular}

\section{DISCUSIÓN}

El presente trabajo supone una aproximación a la evaluación de una estrategia de difusión de la información en salud pública. Se trata de un estudio exploratorio que permite conocer algunos aspectos relacionados con una actividad esencial en la vigilancia epidemiológica y que, por tanto, debería ser evaluada de una forma explícita y rutinaria. Los resultados del estudio indican una buena acogida de la HISP del Área 2 entre los profesionales sanitarios. Esto se manifiesta tanto en el alto porcentaje de los que dicen conocerla como en la valoración que realizan de ella.

Antes de comentar los resultados de la encuesta es necesario valorar algunos aspectos metodológicos. Los resultados obtenidos no parecen estar afectados por un sesgo de selección, ya que las personas de la muestra que han contestado a la encuesta no presentan diferencias con respecto al conjunto de profesionales sanitarios del área. La tasa de respuesta obtenida ha sido alta, sin embargo, en general, ha sido necesario solicitar por dos veces a los encuestados que cumplimentasen el cuestionario. Es conocido que las encuestas por correo suelen obtener una tasa de respuesta baja ${ }^{10}$, esto es lo que ha ocurrido en nuestro caso, a pesar del interés que manifiestan los profesionales por la devolución de información. Sin embargo, el recuerdo telefónico se ha mostrado útil para mejorar la tasa de respuesta ${ }^{11}$. El tipo de encuesta a utilizar (por correo, con entrevistador, etcétera) debe ser considerado en función de las tasas de no respuesta estimadas, el tiempo, el presupuesto y la disponibilidad de otros recursos. No obstante, es imprescindible mantener la evaluación de las estrategias de devolución de información aunque exijan un esfuerzo y un gasto no despreciables. Otro aspecto de interés es el relativo a la medición de los conceptos oportuno, aceptable y útil. El concepto de oportunidad es quizás el más fácil de objetivar. En el caso de un sistema de vigilancia ha sido definida como el «intervalo de tiempo entre la ocurrencia de un evento adverso para la salud y (i) la notificación, (ii) la identificación de la tendencia o el brote, o (iii) el establecimiento de medidas de control» ${ }^{12}$. Para un sistema de devolución de información podríamos definir la oportunidad como «el intervalo de tiempo entre la ocurrencia de un evento de interés en salud pública y la difusión de la información sobre el mismo entre los que deben conocerlo». En nuestro caso esto lo medimos de forma indirecta mediante la percepción que el receptor de la información tiene sobre el retraso con que la recibe, así como por la periodicidad con la que desea obtener la información. El concepto aceptabilidad ha sido siempre difícil de medir al evaluar sistemas de vigilancia ${ }^{12}$, en nuestro caso creemos que el uso de la información ofrecida por la HISP en sesiones de trabajo y la frecuencia de consulta de los 
contenidos de la misma y el interés manifestado por los mismos, constituyen una buena aproximación a este constructo; de forma indirecta la propia tasa de respuesta podría aproximar también esta idea. Finalmente, la utilidad es valorada de forma subjetiva por el propio usuario de la información ${ }^{11}$ en dos componentes, la utilidad para mejorar el conocimiento de la realidad epidemiológica de la zona en la trabajan y la utilidad para la toma de decisiones en su ámbito de trabajo. En el estudio no se valora qué tipo de decisiones pueden tomar cada uno de los profesionales a la luz de la información ofrecida en la HISP, cuestión en la que sería de interés profundizar. A pesar de esto podemos hacer algunas suposiciones sobre las mismas. Así, cuando en la HISP se incluye información sobre un brote de tos ferina, el médico que está atendiendo un caso con tos persistente en la zona puede aumentar su sospecha diagnóstica y pedir las pruebas complementarias necesarias para confirmar o descartar el diagnóstico; cuando en la HISP se incluye información sobre la hipertensión arterial, el personal de enfermería que trabaja en Educación para la Salud, puede adaptar sus mensajes; cuando se informa sobre un seminario, un curso o cualquier otro evento, sirve para decidir acudir o no. Creemos que no es necesario saber exactamente a qué toma decisiones ha ayudado la información ofrecida en la HISP al hacer una evaluación como la que se hace en este trabajo, aunque sería útil de cara al diseño de los contenidos de la misma para optimizar su utilidad en el trabajo diario.

El porcentaje de profesionales que conocen la Hoja ISP es algo menor que el de los que conocen el BECM. Esto se puede explicar por su diferencia de antigüedad, ya que el BECM existe desde hace más 10 años mientras que la HISP está en su primer año. No obstante, de mantenerse en el tiempo, parece razonable pensar que la HISP termine siendo más conocida que el BECM, por ser una herramienta con información más frecuente y más cercana al profesional del Área, ya que trata sobre eventos ocurridos en la misma durante la últi- ma semana. Estas mismas razones son probablemente las que explican que la HISP sea más consultada que el BECM. Respecto a los contenidos de la HISP se observa que, en general, los profesionales valoran más los temas relacionados con situaciones estresantes: las alertas y la enfermedad meningocócica. Por el contrario los contenidos relativos a la temporada de gripe son menos valorados, a pesar de que pueden tener repercusiones importantes en la organización del trabajo. Esto ocurre tanto si se analiza globalmente todo el colectivo de encuestados, como si se hace por separado para médicos y DUE (datos no mostrados). Otro aspecto importante es el hecho de que el 56,6\% de los entrevistados comente los contenidos de la HISP en alguna reunión de equipo, servicio o sesiones clínicas, lo que da una idea de la aceptación de la HISP por los profesionales sanitarios del Área. Además, ello proporciona una presencia de la salud pública en el trabajo diario de los mismos.

Llama la atención que la mayoría de los médicos recibe algún tipo de información de salud pública (el BECM, la HISP o ambos), mientras que los DUE se diferencian en dos categorías, los que reciben información de salud pública, generalmente ambos boletines (BECM y HISP), y los que no reciben ninguna información. Esto indica que el conocimiento de la HISP depende de los circuitos de difusión de la información de salud pública y que éstos funcionan de forma desigual entre los sanitarios asistenciales. Explica también que el factor que más influye en el conocimiento de la HISP sea el conocimiento del BECM. Vemos, pues, que una proporción importante de DUE, en los que recae buena parte de las actividades de educación sanitaria y muchas consultas sobre problemas de salud, carecen de un sistema que les acerque a la realidad epidemiológica y de salud pública de la población a la que atienden. Esta necesidad es claramente percibida por ellos mismos y expresada a través de las sugerencias recogidas en el cuestionario (resultados no presentados en este trabajo). 
Se puede concluir que esta investigación, aunque se trate de una aproximación exploratoria a una estrategia de difusión de información en salud pública, permite identificar algunos puntos de crecimiento. Es necesario mejorar la difusión de la información de salud pública entre los profesionales no médicos. La información ofrecida, aunque es bien valorada en conjunto, debe evolucionar de modo que permita su uso para una toma de decisiones más informada. Creemos que resultaría de interés el desarrollo de la metodología de evaluación de este tipo de sistemas, de forma que además permitieran la comparación entre distintas estrategias. Finalmente, parece que la periodicidad adecuada para la HISP es la semanal. A pesar del importante esfuerzo técnico y humano que supone su edición, esa periodicidad la dota de una agilidad y una facultad de comunicación capaces de proporcionar a «todos los que deben conocerla» información oportuna para la toma de decisiones ${ }^{1}$.

\section{BIBLIOGRAFÍA}

1. Langmuir AD. The Surveillance of Communicable diseases of national importance. $\mathrm{N}$ Engl J Med 1963; 268:182-91.

2. Fossaert H, Llopis A, Tigre CH. Sistemas de Vigilancia Epidemiológica. Bol Oficina Sanit Panam 1974;76:512-28.

3. Thacker SB, Stroup DF. Future Directions for Comprehensive Public Heatlh Surveillance and Health Information Systems in the United States. Am J Epidemiol 1994;140:383-97.

4. Editorial. 10 años de existencia del Boletín Epidemiológico de la Comunidad de Madrid. Bol Epidemiol Comunidad Madrid 1999;6:3.
5. Fernández Merino JC, Maceiras Rodríguez L, Zurriaga Lloréns O. La discusión electrónica o qué son las listas de distribución. Gac Sanit 2000; $14: 314-317$

6. Klaucke DN, Buehler JW, Thacker SB, Gibson Parrish R, Trowbrigde FL, Berkelman RL, et al. Guidelines for evaluating surveillance systems. MMWR 1998; 37(S-5):1-8.

7. Kimball AM, Thacker SB, Levy ME. Shigella surveillance in a large metropolitan area: assessment of a passive reporting system. Am J Public Health 1980;70:164-6.

8. Vogt RL, Larue D, Klaucke DN, Jillson DA Comparison of active and passive surveillance systems of primary care providers for hepatitis, measles, rubella and salmonellosis in Vermont. Am J Public Health 1983;73:795-7.

9. Segura del Pozo J, Martínez Navarro F. Evaluación de la vigilancia epidemiológica de infección meningocócica en las Áreas III y V de la Comunidad de Madrid durante el período 1994-1996. Bol Epidemiol Sem 1997; 5:225-32.

10. McColl E, Jacoby A, Thomas L, Soutter J, Bamford C, Steen N et al. Design and use of questionnaires: a review of best practice applicable to surveys of health service staff and patients. Health Technol Assess 2001; 5:1-256.

11. Salim Silva M, Smith WT, Bammer G. Telephone reminders are cost effective in improving responses to postal surveys. J Epidemiol Community Health $2002 ; 56: 115-8$.

12. Thacker SB, Parrish RG, Trowbridge FL and Surveillance Coordination Group. A method for evaluating systems of epidemiological surveillance. World Health Stat Q. 1988;41(1):11-8.

13. Barclay S, Todd C, Finlay I, Grande G, Wyatt P. Not another questionnaire! Maximizing the response rate, predicting non-response and assessing non-response bias in postal questionnaire studies of GPs. Fam Pract 2002;19:105-111. 